\title{
The ERLIN1-CHUK-CWF19L1 gene cluster influences liver fat deposition and hepatic inflammation in the NHLBI Family Heart Study
}

\author{
Mary F. Feitosa ${ }^{\mathrm{a},{ }^{\star},}$, Mary K. Wojczynski ${ }^{\mathrm{a}}$, Kari E. North ${ }^{\mathrm{b}}$, Qunyuan Zhang ${ }^{\mathrm{a}}$, Michael A. \\ Province $^{\mathrm{a}}$, Jeffrey J. Carr ${ }^{\mathrm{C}}$, and Ingrid B. Borecki ${ }^{\mathrm{a}}$ \\ aDepartment of Genetics, Washington University School of Medicine \\ bDepartment of Epidemiology and Carolina Center for Genome Sciences, University of North \\ Carolina at Chapel Hill \\ 'Translational Science Institute, Wake Forest University School of Medicine
}

\section{Abstract}

Objectives-Nonalcoholic fatty liver disease (NAFLD) ranges from simple steatosis to hepatic inflammation to cirrhosis. We sought to identify common genetic variants contributing to NAFLD, using CT measured fatty liver (FL), and alanine aminotransferase levels (ALT), as a biochemical marker of hepatic inflammation.

\begin{abstract}
Methods-We employed a correlated meta-analysis (CMA) to test whether combining FL and ALT genomewide association (GWA) results, using 2.5 million imputed SNPs, could enhance ability to detect variants influencing both traits.

Results-Variants of the ERLIN1-CHUK-CWF19L1 gene cluster were associated with concomitant variation of FL and ALT. Nine variants (rs2862954, rs1408579, rs10883451, rs11597086, rs11591741, rs17729876, rs17668255, rs17668357, rs12784396) displayed genomewide significant associations at loci concomitantly influencing FL and ALT $\left(2.47 \times 10^{-9} \leq \mathrm{CMA}-\mathrm{p} \leq 4.29 \times 10^{-10}\right)$ as compared with the suggestive significance of marginal tests $\left(4.11 \times 10^{-5} \leq \mathrm{GWA}-\mathrm{p} \leq .34 \times 10^{-6}\right)$. For example, the missense variant in ERLIN1-rs2862954 was genomewide significant (CMA-p $\left.=4.88 \times 10^{-10}\right)$ for the combination of FL and ALT, while the respective univariate associations were suggestive $\left(\mathrm{FL}: \mathrm{p}=5.74 \times 10^{-6}\right.$, ALT:p $\left.=3.71 \times 10^{-6}\right)$. Further we investigated whether the concomitant associations were driven mainly by ALT levels. When we adjusted FL by ALT, the correlated associations diminished but did not vanish (CMAp $3.3 \times 10^{-7}$ ). Our findings suggest ERLIN1-CHUK-CWF19L1 variants are associated with early stage of FL accumulation (measured by CT) to hepatic inflammation (ALT levels), and the association enhances when accounting for the correlations between their scans.
\end{abstract}

Conclusions-CMA approach enhanced the ability to identify novel variants of the ERLIN1CHUK-CWF19L1 influencing both simple steatosis and hepatic steatosis with inflammation,

(C) 2013 Elsevier Ireland Ltd. All rights reserved.

*Correspondent Author: Mary Furlan Feitosa, Ph.D., Department of Genetics, Washington University School of Medicine, 4444 Forest Park Blvd; St. Louis, MO, 63108-2212, Campus Box 8506, USA, Phone: (314) 747.3792; Fax: (314) 362.4227; mfeitosa@wustl.edu. Conflict of interest

None.

Publisher's Disclaimer: This is a PDF file of an unedited manuscript that has been accepted for publication. As a service to our customers we are providing this early version of the manuscript. The manuscript will undergo copyediting, typesetting, and review of the resulting proof before it is published in its final citable form. Please note that during the production process errors may be discovered which could affect the content, and all legal disclaimers that apply to the journal pertain. 
which suggest that this gene cluster may regulate the susceptibility of NAFLD in a wide spectrum of disease.

\section{Introduction}

Nonalcoholic fatty liver disease (NAFLD) is an emerging metabolic-related disorder characterized by hepatic fat accumulation, predominantly in the form of triglycerides (TG), in the absence of significant ethanol consumption or other known causes of liver disease [1, 2]. NAFLD is a common disease and ranges from simple steatosis, which is associated with metabolic abnormalities, to nonalcoholic steatohepatitis (NASH), which presents as inflammatory damage in the liver and can progress ultimately to cirrhosis and liver cancer [1].

Computed tomography (CT) imaging of the liver is a non-invasive procedure which allows for an accurate and reliable assessment of fat content in the hepatic parenchyma. There is an inverse relationship between liver fat burden and liver attenuation, and it has a sensitivity of $82 \%$ and $100 \%$ specificity for detecting $>30 \%$ steatosis $[3,4]$. Alanine transaminase (ALT) is a liver-derived enzyme, and elevated serum levels of ALT are associated with liver damage. ALT has been used as a biochemical marker of hepatic inflammation to monitor the course and severity of NAFLD [5, 6], although it is a non-specific marker of liver damage.

The heritabilities of these traits have been estimated as $25 \%-30 \%$ for CT measured fatty liver (FL [7]), and 33\% - 55\% for serum ALT levels [8, 9]. To date, a limited number of genes has demonstrated associations with susceptibility to FL, including PNPLA3, NCAN, $P P P 1 R 3 B, G C K R$, and LYPLAL1, and explain less than $5 \%$ of the proportion of variance in FL [7, 10-13]. Also, association of CPN1-ERLIN1-CHUK with ALT levels was reported [8]. There is a significant correlation between these traits of NAFLD. The correlation between FL an ALT was reported as 0.405 [14], suggesting common genetic effect. Simultaneous consideration of FL and ALT could enhance the identification of etiologic factors, especially genes. The goal of this study was to search for genetic variants that account for some of the correlated architecture between CT measured FL and levels of ALT by combining the genomewide association scans for these phenotypes in a correlated metaanalysis (CMA) using 2,705 European American participants of the NHLBI Family Heart Study (FamHS).

\section{Material and Methods}

\subsection{Study Design and Sample}

The FamHS (https://dsgweb.wustl.edu/fhscc/) recruited 1,200 families, half randomly sampled, and half selected because of an excess of coronary heart disease or related-risk factor abnormalities as compared with age- and sex-specific population rates [15] from four population-based parent studies: the Framingham Heart Study, the Utah Family Tree Study, and two centers for the Atherosclerosis Risk in Communities study (Minneapolis, and Forsyth County, NC). Study participants belonging to the largest pedigrees were invited to undergo CT imaging for assessment of coronary artery calcification; these scans also were used to quantitate hepatic and abdominal fat burden. A total of 2,767 Caucasian subjects in 510 extended families were examined.

\subsection{Phenotypes}

Participants underwent a cardiac multidetector CT exam with four detectors using a standardized protocol as described previously [16]. In brief, two cardiac gated images of the thorax included the upper third of the liver. The unenhanced CT images were reviewed on a GE Advantage Windows Workstation (General Electric Healthcare, Waukesha, WI). Liver 
attenuation (LA) was measured by placing three round regions of interest (ROI's) in two slices of the superior right lobe of the liver. The six ROI's values (three from each slice) were then averaged as a mean LA. All participants were imaged with standardized phantom that contains material that simulates water $(\mathrm{HU}=0)$ as well as increasing densities of calcium (50,100 and $200 \mathrm{mg}$ of calcium). The phantom measures at water density were used to adjust and standardize LA measurements. Lower LA values indicate higher fat content. We inverted the scale of LA by multiplying each value by negative one to produce a phenotype of fatty liver (FL) for which higher values are associated with greater liver fat. ALT (in units of U/L) was measured by reaction rate assay based on the conversion of NADH to NAD using the Vitros analyzer (Johnson \& Johnson Clinical Diagnostics, Inc. Rochester NY 14650).

Subjects were excluded from the analyses if they were: (i) heavy drinkers (men: $>21$ drinks/ week and women: >14 drink drinks/week) or (ii) taking Amiodarone, or (iii) positive for hepatitis C antibodies for ALT analysis (88 subjects for FL and 124 subjects for ALT excluded). Alcohol intake was assessed during interview. The subjects reported whether he/ she "presently drinks alcoholic beverage at all (alcohol intake: $0=$ No, $1=$ Yes)" and if so, the number of $1.5 \mathrm{oz}$ cocktails, $12 \mathrm{oz}$ glasses (or can) of beer, and $4 \mathrm{oz}$ glasses of wine they consumed per day in 1 week. Total number of alcohol intake in grams per day was calculated from the reported intakes of beer, wine, and spirits. Medications were assessed at the clinic visit in which subjects brought all medications they were taking. Amiodarone has the effect of increasing liver density, and thus we excluded subjects taking this medication. Finally, information of hepatitis $\mathrm{C}$ antibodies was obtained from questionnaire.

\subsection{Genotypes}

A total of 2,705 subjects for the analysis were typed on three Illumina platforms: (i) 974 subjects with 547,353 SNP markers on the Illumina HumMap 550K chip, (ii) 249 subjects with 576,888 SNP markers on the Human 610-Quadv1 Illumina chip, and (iii) 1,482 subjects with 1,111,639 SNP markers on the Human 1M-Duov3 Illumina chip [18]. Quality control was performed before imputation by checking pedigree relationships (using GRR software), and excluding SNPs with low call rates $(<98 \%)$. A framework map for imputation was defined by choosing SNPs on all platforms, and restricting to those with minor allele frequency between $1 \%$ and 50\%, and excluding SNPs with significant $(\mathrm{p}<1.0 \times$ $10^{-6}$ ) departure from Hardy-Weinberg equilibrium. This panel was used to impute $\sim 2.5 \mathrm{M}$ SNPs based on phased haplotypes from HapMap (release 22, build 36, CEU, http:// hapmap.ncbi.nlm.nih.gov/downloads/phasing/2007-08_rel22/phased/) using MACH (version 1.0.16, http://www.sph.umich.edu/csg/abecasis/mach/). The final data for the GWA analysis included 2,584 participants for ALT levels and 2,597 participants for CT measured FL.

\subsection{Statistical Analysis}

2.4.1. Covariate adjustment-Principal components (PC [19]) were estimated using the genotypes of the largest sample of independent subjects $(\mathrm{N}=753)$ and then calculated for family members. The phenotypes were adjusted using two steps: (1) forcing field centers and genotype chip effects into the regression analysis, and then (2) adding age, age ${ }^{2}$, sex, current alcohol intake (Yes/No), phantom, and ten PC using stepwise regression analysis and retaining terms significant at the 5\% level. The residuals were standardized to a mean of 0 and variance of 1 (FL and ALT). Finally, an inverse normal rank transformed within sex was performed for FL. In the second adjustment for FL, ALT was forced in the covariate list (in step 1); while FL was forced in the covariate list for ALT (in step 1), similarly to the first adjustment procedure. 
2.4.2. Heritability estimates-The heritabilities were calculated using a variance components model as implemented in the computer program SOLAR (http://txbiomed.org/ departments/genetics/genetics-detail?P=37).

2.4.3. Association analysis-GWA analysis was first carried out for each of ALT and FL assuming additive effects with mixed model linear regression, accounting for dependency among family members as a function of their kinship correlations (R kinship package: http://cran.r-project.org/web/packages/GWAF/). Results were filtered to include SNPs with acceptable imputation quality $\left(r^{2}\right.$ hat $\left.>0.3\right)$ and with effect allele frequency between 1-99\%. To test whether individual variants influence both FL and ALT, we employed a novel correlated meta-analysis (CMA) approach [20], which combines the SNPwise Z-scores corresponding to the p-values, while accounting for the correlation between scans. In brief, the standard meta-analysis assumes all component scans are independent; however, correlations among scans can arise for a number of reasons, here, mainly because the same set of subjects was used for both phenotypes and subjects' phenotypes are correlated. Province and Borecki [20, 21] developed a method to empirically estimate the covariance among scans to be included in a meta-analysis, and to correct the signal inference in the combined meta p-value. Briefly, all p-values are first converted to corresponding zscores, which are assumed to follow a multivariate normal distribution. Since the vast majority of SNPs in a genome scan is under the null for any given phenotype, the global degree of correlation between the scans can be calculated to assess the degree of nonindependence. A tetrachoric correlation estimator is used on p-values dichotomized as $(\mathrm{P} \unlhd 0.5 ; \mathrm{P}>0.5$ ) to minimize the effect of the few SNPs that might be under the alternative. The estimated correlation is used to adjust the variance estimate of the combined Z-scores.

2.4.3. Regional plot-It was generated with LocusZoom [22], and Haploview (http:// haploview.software.informer.com/) was used for investigation of linkage disequilibrium (LD) and block structure using the HapMap CEU data.

\section{Results}

\subsection{Characteristics of the Study Sample}

Table 1 shows the characteristics of the FamHS participants included in the analysis, in which $56 \%$ of subjects were women. The average age was 57 years, and average body mass index (BMI) was $28.9 \mathrm{~kg} / \mathrm{m}^{2}$. The proportion of subjects with severe obesity (BMI $\geq 35.0$ $\left(\mathrm{kg} / \mathrm{m}^{2}\right)$ was $12.5 \%$ and with diabetes was $13.4 \%$. The mean levels of TG $(144.1 \pm 93.9 \mathrm{mg} /$ $\mathrm{dL})$, ALT (21.7 $\pm 15.8 \mathrm{U} / \mathrm{L}[6])$ and LA $(59.1 \pm 11.2 \mathrm{HU}[3])$ were within normal range values. The phenotypic correlation between FL and ALT levels was $0.27(\mathrm{p}<0.0001)$. The heritability estimates were significant $(\mathrm{p}<0.0001$ ) for the covariate-adjusted phenotypes, FL $(0.27 \pm 0.04)$ and ALT $(0.26 \pm 0.04)$, suggesting that genetic factors influence the variability levels of these traits and a search for significant genetic variants is warranted.

\subsection{Genomewide association analyses}

Univariate GWA analysis revealed associated variants near or in PNPLA3 and PPP1R3B at genomewide significance levels ( $\mathrm{p}<5.0 \times 10^{-8}$, Supplemental Table 1$)$ in FamHS. The highest association was with a missense locus in PNPLA3 (rs738409: $\beta=0.34$ for the $\mathrm{G}$ allele, $P=2.09 \times 10^{-23}$ ), which was previously reported [7] and also detected for magnetic resonance assessed steatosis [10]. The rs2126259 near- $P P P 1 R 3 B$ associated with FL ( $\beta=$ -0.26 for the A allele, $P=3.28 \times 10^{-8}$ ) is in complete linkage disequilibrium (LD) with rs $4240624\left(\mathrm{LD}=1, \mathrm{r}^{2}=0.80, P=4.76 \times 10^{-8}\right)$, which was also previously reported associated with FL [7]. No loci were associated with ALT levels at genomewide significance level. 
To identify additional variants influencing both FL and ALT, we carried out a correlated meta-analysis of the two individual scans. Largely, the FL and ALT scans provided independent information ( $\mathrm{r}=0.045$ between the scan $\mathrm{p}$-values). This approach yielded evidence for association on 10q24-q25 with variants in ERLIN1-CHUK-CWF19L1 (endoplasmic reticulum (ER) lipid raft associated 1; conserved helix-loop-helix ubiquitous kinase; CWF19-like 1 cell cycle control; and biogenesis of lysosomal organelles complex-1 subunit 2, respectively) gene cluster. The regional plot depicts correlated meta-analysis associations between variants in ERLIN1-CHUK-CWF19L1 gene cluster with FL and ALT (Figure 1). Nine SNPs located in two haplotype blocks (HB-1: rs2862954, rs1408579 and rs10883451 in ERLIN1; and HB-2: rs11597086 and rs11591741 in CHUK, and rs17729876, rs17668255, rs17668357 and rs12784396 in CWF19L1, Supplementary Figure 1) were strongly associated with concomitant variation in FL and ALT levels $\left(4.29 \times 10^{-10} \leq\right.$ CMA-p $\leq 1.84 \times 10^{-9}$ ). For example, the correlated meta-analysis showed evidence of a novel association between the missense-ERLIN1-rs2862954 substitution $\mathrm{C}>\mathrm{T}$ with decreasing levels of both FL ( $\beta=-0.13 \pm 0.03$, C allele frequency $=0.48)$ and $\operatorname{ALT}(\beta=-0.14 \pm 0.03$, Table 2). This finding suggests that the missense-ERLIN1-rs2862954 may regulate the susceptibility of NAFLD in a broad spectrum of disease. Thus, the correlated meta-analysis approach enhanced the ability to detect association at genomewide significance level as compared with suggestive individual scans $\left(2.71 \times 10^{-6} \leq \mathrm{GWA}-\mathrm{p} \leq 2.75 \times 10^{-5}\right.$ for FL or ALT, Table 2). In addition to these nine SNPs, nineteen SNPs within CHUK-CWF19L1 or near-ERLIN1 genes showed suggestive associations with concomitant variation of FL and ALT levels (CMA-p $<1.0 \times 10^{-6}$, Supplementary Table 2, Supplementary Figure 1).

Since diabetic status and severe obesity are metabolic-related disorders with NAFLD and may represent potential confounders with NAFLD, we tested the robustness of these associations while restricting the analysis to subgroups of subjects free of diabetes and/or severe obesity (BMI $\geq 35.0 \mathrm{~kg} / \mathrm{m}^{2}$ ). Even though the sample sizes were reduced $\sim 23 \%$ after excluding diabetics and severe obese (ALT: 2,597 vs. 2,008 subjects and CT measured FL: 2,584 vs. 1,995 subjects), the association results for CMA and univariate analyses remained similar (Supplementary Table 3A). Also, the results were similar in non-diabetics (Supplementary Table 3B) and in non-extreme obese subjects (Supplementary Table 3C). These findings show evidence that variants within ERLIN1-CHUK-CWF19L1 gene cluster have concomitant effects on NAFLD traits, even after accounting for some components related to metabolic syndrome (e.g., diabetes and severe obesity).

To test if the correlated associations of ERLIN1-CHUK-CWF19L1 variants with NAFLD traits were predominantly due to ALT levels, we adjusted LA by ALT (in addition to the other covariates, LA_ALT) and carried out correlated meta-analysis between ALT and FL_ALT. The genomewide significance levels of the correlated meta-analysis were slightly attenuated (CMA-p $<3.3 \times 10^{-7}$, Supplementary Table 4A). We also found similar pattern of correlated meta-analysis associations when we used the adjusted ALT by FL (ALT_FL) and FL (CMA-p $<3.7 \times 10^{-8}$, Supplementary Table 4B). These findings suggest that variants of ERLIN1-CHUK-CWF19L1 gene cluster influence both NAFLD traits (FL and ALT), and the significance of association enhances when accounting for the correlation between their scans.

\section{Discussion}

Variants of the ERLIN1-CHUK-CWF19L1 gene cluster associated with both FL and ALT levels were identified by correlated meta-analysis. Among the nine SNPs at genomewide significance levels, three of them were in one ERLIN1 haplotype block (rs2862954, rs1408579 and rs10883451), which includes the missense-ERLIN1-rs2862954 associated with lower levels of FL and ALT. The other six SNPs were in a single haplotype block that 
spans $C H U K$ (rs11597086 and rs11591741) and CWF19L1 (rs17729876, rs17668255, rs17668357 and rs12784396). In addition, our findings show that these common variants may influence both FL and ALT levels, even after accounting for some components related to metabolic syndrome, such as extreme obesity and diabetes.

Association of missense-ERLIN1-rs $2862954\left(\mathrm{p}=9.24 \times 10^{-8}\right.$ [8]) was previously reported with ALT levels in a genomewide meta-analysis study including 12,419 European white subjects (the CoLaus Study from Lausanne Switzerland, the InCHIANTI Study from Tuscany Italy, and the LOLIPOP Study from London UK). ALT levels have been used as a biochemical marker of hepatic inflammation [5, 6]; however it is a non-specific marker of liver damage. Our study shows for the first time that missense-ERLIN1-rs2862954 is relevant for the early stage of FL accumulation, even before liver damage occurs. ERLIN1 encodes a member of the prohibitin family of proteins that define lipid-raft-like domains of the endoplasmic reticulum [23]. Lipid rafts are regions of membranes with a distinct, characteristic structural composition and that appear to act as platforms to colocalize proteins involved in intracellular signaling pathways [24]. ERLIN1 is expressed in the liver, among other tissues [25].

Variants of $C H U K$ were also previously reported associated with ALT levels (rs11591741: $\mathrm{p}=2.97 \times 10^{-8}$ and $\mathrm{rs} 11597086: \mathrm{p}=1.83 \times 10^{-8}[8]$ ), and NASH fibrosis (rs11591741: $\mathrm{p}=0.010$ [13]) in a multivariate model that accounted for sex, BMI, diabetes, alcohol consumption and PNPLA3 variant (rs738409). The 894 adult patients from the NASH Clinical Research Network and National Institutes of Health Clinical Center studies were predominantly Caucasian $(85.7 \%$ ) and $59.4 \%$ had definite histological diagnosis for NASH [13]. CHUK-intronic-rs11591741 is in complete LD with the $5^{\prime}$ utr-CWF19L1-rs12784396 $\left(\mathrm{LD}=1\right.$ and $\left.\mathrm{r}^{2}=1\right)$. These findings suggest a regulatory effect on transcription of $C H U K$ $C W F 19 L 1$ that may influence the development and severity of NAFLD. The CHUK gene encodes a protein that is an inhibitor of the essential transcription nuclear factor-kappa-beta (NF- $\mathrm{KB}$ ) complex via ubiquination pathway, and is involved in inflammatory responses triggered by cytokines [6]. It is worth pointing out that NF-B activated by IKK-B (IKB kinase) is implicated in transcription of proinflammatory mediators (e.g., interleukin-1 (IL-1), interleukin-6 (IL-6) and TNF-a). NF-B dependent inflammatory mediators produced in hepatocytes down-regulate insulin sensitivity and trigger liver injury [26, 27], and is often elevated in patients with NASH $[1,27]$. The $C W F 19 L 1$ gene is conserved in several species; however its function remains unknown.

The $S C D$ gene (10q24.31), located $79 \mathrm{~kb}$ downstream from the $5^{\prime}$ utr- $C W F 19 L 1$ rs 12784396, was previously reported influence NAFLD in mice and in humans. SCD encodes the stearoyl-CoA desaturase 1 (SCD1) and is involved in both the lipogenic as well as the lipid oxidative pathways [28]. SCD1 is a central enzyme in lipid metabolism; it converts saturated fatty acids to monounsaturated fatty acids, which are major substrates for synthesis of triacylglycerols and other lipids [28]. SCD1 knockout mice were resistant to the development of obesity and hepatic steatosis [28], whereas the activity of SCD1 was significantly increased in the fatty livers of $o b / o b$ mice. In addition, hepatic SCD1 activity was increased in nonalcoholic human fatty liver [29], and associated with fatty liver and insulin resistance in obese humans [30]. Although $S C D$ is a plausible candidate gene for NAFLD, we could not detect association of variants within the $S C D$ gene with NAFLD traits (FL and ALT) nor has any other study found such an association, as far as we know. However, as it was previously suggested in mice, the activation of the transcription of SCD1 may occur via the numerous NF-B elements in the SCD1 promoter [28], and/or occur via endoplasmic reticulum stress and inflammatory response mRNAs [31]. If there is a pathway linking activity of SCD1 and CHUK protein-inhibitor NF- $\mathrm{kB}$, and/or activity of SCD1 and 
ERLIN-1 protein of lipid-raft-like domain are unknown, and further studies will be necessary to prove or disprove these hypotheses in human.

Presently, it is not possible to accurately predict which subjects with simple steatosis will develop to steatohepatitis and fibrosis, and much less is known about genetic variants influencing the progression of NAFLD. These findings suggest that variants within the ERLIN1-CHUK-CWF19L1 gene cluster contribute to the increased susceptibility to simple steatosis (current FamHS results), hepatic injury (ALT [8], and the current FamHS results) and NAFLD histological severity (fibrosis [13]). It could be plausible that the evolution of NAFLD, from simple steatosis to hepatic inflammation to fibrosis, is due to the critical accumulation of liver fat, and they do not represent separate effects. Further studies will be necessary to understand the diagnosis and progression of NAFLD. Furthermore, our metaanalysis approach is the first study to report a correlated association between ERLIN1CHUK-CWF19L1 variants with the liver fat content and serum plasma ALT levels. Although our study shows evidence that variants of this gene cluster confer increased susceptibility to a broad spectrum of NAFLD, additional investigations will be necessary to understand how genetic effects of ERLIN1-CHUK-CWF19L1 contribute to individual susceptibility to simple steatosis to the development of hepatic inflammation.

Our study has some limitations but several relevant strengths. Some of the strengths include: ALT levels are known as a sensitive screening test in detecting liver disease better than AST (aspartate aminotransferase) or other biochemical markers [32], and are strongly correlated with biopsy-proven fatty liver disease [6]. ALT levels have also been used to define NAFLD in epidemiological studies. Fatty liver accumulation was directly measured by CT liver attenuation, which is a non-invasive procedure that provides an accurate characterization of fat burden [3, 4]. Our novel correlated meta-analysis approach also provided an objective method to screen variants influencing correlated phenotypes, which resulted in associations that implicate novel variants (e.g., rs12784396 in the utr-5 CWF19L1) for NAFLD. Also, some SNPs that we found associations with concomitant variations of FL and ALT were previously reported associations with ALT levels [8] and with histological fibrosis [13]. The evidence of association between missense-ERLIN1-rs2862954 with NAFLD traits warrant functional followup studies. A limitation of our study is that ALT levels and CT measured FL do not provide the clinical precision of diagnostic forms of liver disease based on histology, which would require biopsies. However, we excluded subjects with high alcohol intake and hepatitis $\mathrm{C}$ positive to reduce this possible source of heterogeneity. Moreover, both CT measured FL and ALT levels bring opportunity to study NAFLD.

In brief, our study identified common variants of the ERLIN1-CHUK-CWF19L1 gene cluster associated with NAFLD in a wide spectrum, simple steatosis, as CT measured FL, and hepatic inflammation measured by plasma levels of ALT. The discovery of these genetic variants may aid in understanding the correlated architecture between liver fat deposition and inflammation process, NAFLD development, and the prospective risk factors including type 2 diabetes and cardiovascular disease.

\title{
Supplementary Material
}

Refer to Web version on PubMed Central for supplementary material.

\section{Acknowledgments}

\author{
Sources of Funding
}

This study was supported by the by NIH grants R01-HL-087700 and R01-HL-088215 (Michael A. Province, PI) from NHLBI; and R01-DK-8925601 and R01-DK-075681 (Ingrid B. Borecki, PI) from NIDDK. 


\section{References}

1. Lewis JR, Mohanty SR. Nonalcoholic fatty liver disease: a review and update. Dig Dis Sci. 2010; 55:560-578. [PubMed: 20101463]

2. Musso G, Gambino R, Cassader M. Recent insights into hepatic lipid metabolism in non-alcoholic fatty liver disease (NAFLD). Prog Lipid Res. 2009; 48:1-26. [PubMed: 18824034]

3. Schwenzer NF, Springer F, Schraml C, Stefan N, Machann J, Schick F. Non-invasive assessment and quantification of liver steatosis by ultrasound, computed tomography and magnetic resonance. $\mathrm{J}$ Hepatol. 2009; 51:433-445. [PubMed: 19604596]

4. Boyce CJ, Pickhardt PJ, Kim DH, et al. Hepatic steatosis (fatty liver disease) in asymptomatic adults identified by unenhanced low-dose CT. AJR Am J Roentgenol. 2010; 194:623-628. [PubMed: 20173137]

5. Yamada J, Tomiyama H, Yambe M, et al. Elevated serum levels of alanine aminotransferase and gamma glutamyltransferase are markers of inflammation and oxidative stress independent of the metabolic syndrome. Atherosclerosis. 2006; 189:198-205. [PubMed: 16405892]

6. Schindhelm RK, Diamant M, Dekker JM, Tushuizen ME, Teerlink T, Heine RJ. Alanine aminotransferase as a marker of non-alcoholic fatty liver disease in relation to type 2 diabetes mellitus and cardiovascular disease. Diabetes Metab Res Rev. 2006; 22:437-443. [PubMed: 16832839]

7. Speliotes EK, Yerges-Armstrong LM, Wu J, et al. Genomewide association analysis identifies variants associated with nonalcoholic fatty liver disease that have distinct effects on metabolic traits. PLoS Genet. 2011; 7:e1001324. [PubMed: 21423719]

8. Yuan X, Waterworth D, Perry JR, et al. Population-based genomewide association studies reveal six loci influencing plasma levels of liver enzymes. Am J Hum Genet. 2008; 83:520-528. [PubMed: 18940312]

9. Makkonen J, Pietiläinen KH, Rissanen A, Kaprio J, Yki-Järvinen H. Genetic factors contribute to variation in serum alanine aminotransferase activity independent of obesity and alcohol: a study in monozygotic and dizygotic twins. J Hepatol. 2009; 50:1035-1042. [PubMed: 19303161]

10. Romeo S, Kozlitina J, Xing C, et al. Genetic variation in PNPLA3 confers susceptibility to nonalcoholic fatty liver disease. Nat Genet. 2008; 40:1461-1465. [PubMed: 18820647]

11. Kotronen A, Johansson LE, Johansson LM, et al. A common variant in PNPLA3, which encodes adiponutrin, is associated with liver fat content in humans. Diabetologia. 2009; 52:1056-60. [PubMed: 19224197]

12. Sookoian S, Castaño GO, Burgueño AL, Gianotti TF, Rosselli MS, Pirola CJ. A nonsynonymous gene variant in the adiponutrin gene is associated with nonalcoholic fatty liver disease severity. $\mathrm{J}$ Lipid Res. 2009; 50:2111-2116. [PubMed: 19738004]

13. Rotman Y, Koh C, Zmuda JM, Kleiner DE, Liang TJ, NASH CRN. The association of genetic variability in patatin-like phospholipase domain-containing protein 3 (PNPLA3) with histological severity of nonalcoholic fatty liver disease. Hepatology. 2010; 52:894-903. [PubMed: 20684021]

14. Devries MC, Samjoo IA, Hamadeh MJ, Tarnopolsky MA. Effect of endurance exercise on hepatic lipid content, enzymes, and adiposity in men and women. Obesity (Silver Spring). 2008; 16:22812288. [PubMed: 18719669]

15. Higgins M, Province M, Heiss G, et al. NHLBI Family Heart Study: objectives and design. Am J Epidemiol. 1996; 143:1219-1228. [PubMed: 8651220]

16. Carr JJ, Nelson JC, Wong ND, et al. Calcified coronary artery plaque measurement with cardiac CT in population-based studies: standardized protocol of Multi-Ethnic Study of Atherosclerosis (MESA) and Coronary Artery Risk Development in Young Adults (CARDIA) study. Radiology. 2005; 234:35-43. [PubMed: 15618373]

17. Giannini EG, Testa R, Savarino V. Liver enzyme alteration: a guide for clinicians. CMAJ. 2005; 172:367-379. [PubMed: 15684121]

18. Kraja AT, Vaidya D, Pankow JS, et al. A bivariate genomewide approach to metabolic syndrome: STAMPEED consortium. Diabetes. 2011; 60:1329-1339. [PubMed: 21386085] 
19. Price AL, Patterson NJ, Plenge RM, Weinblatt ME, Shadick NA, Reich D. Principal components analysis corrects for stratification in genomewide association studies. Nat Genet. 2006; 38:904909. [PubMed: 16862161]

20. Province, MA.; Borecki, IB. A correlated meta-analysis strategy for data mining "OMIC" scans. PSB 2013 Online Proceedings; (on press: http://psb.stanford.edu/psb-online/proceedings/psb13/)

21. Borecki IB, Province MA. Genetic and genomic discovery using family studies. Circulation. 2008; 118:1057-1063. [PubMed: 18765388]

22. Pruim RJ, Welch RP, Sanna S, et al. LocusZoom: regional visualization of genomewide association scan results. Bioinformatics. 2010; 26:2336-2337. [PubMed: 20634204]

23. Browman DT, Resek ME, Zajchowski LD, Robbins SM. Erlin-1 and erlin-2 are novel members of the prohibitin family of proteins that define lipid-raft-like domains of the ER. J Cell Sci. 2006; 119:3149-3160. [PubMed: 16835267]

24. Calder PC, Yaqoob P. Lipid rafts--composition, characterization, and controversies. J Nutr. 2007; 137:545-547. [PubMed: 17311937]

25. Li N, Huang X, Zhao Z, Chen G, Zhang W, Cao X. Identification and characterization of a novel gene KE04 differentially expressed by activated human dendritic cells. Biochem Biophys Res Commun. 2000; 279:487-493. [PubMed: 11118313]

26. Nseir W, Shalata A, Marmor A, Assy N. Mechanisms Linking Nonalcoholic Fatty Liver Disease with Coronary Artery Disease. Dig Dis Sci. 2011; 56:3439-3449. [PubMed: 21655948]

27. Malaguarnera M, Di Rosa M, Nicoletti F, Malaguarnera L. Molecular mechanisms involved in NAFLD progression. J Mol Med. 2009; 87:679-695. [PubMed: 19352614]

28. Flowers MT, Ntambi JM. Role of stearoyl-coenzyme A desaturase in regulating lipid metabolism. Curr Opin Lipidol. 2008; 19:248-256. [PubMed: 18460915]

29. Kotronen A, Seppänen-Laakso T, Westerbacka J, et al. Hepatic stearoyl-CoA desaturase (SCD)-1 activity and diacylglycerol but not ceramide concentrations are increased in the nonalcoholic human fatty liver. Diabetes. 2009; 58:203-208. [PubMed: 18952834]

30. Stefan N, Peter A, Cegan A, et al. Low hepatic stearoyl-CoA desaturase 1 activity is associated with fatty liver and insulin resistance in obese humans. Diabetologia. 2008; 51:648-656. [PubMed: 18286258]

31. Flowers MT, Keller MP, Choi Y, et al. Liver gene expression analysis reveals endoplasmic reticulum stress and metabolic dysfunction in SCD1-deficient mice fed a very low-fat diet. Physiol Genomics. 2008; 33:361-372. [PubMed: 18381840]

32. Ciba I, Widhalm K. The association between non-alcoholic fatty liver disease and insulin resistance in 20 obese children and adolescents. Acta Paediatr. 2007; 96:109-112. [PubMed: 17187615] 


\section{Research highlights}

We employed a novel correlated meta-analysis to improve the power to detect variants associated with NAFLD.

NAFLD was assessed by using CT measured fatty liver and alanine aminotransferase levels (ALT).

Correlated meta-analysis allowed to identify variants of the ERLIN1-CHUKCWF19L1 associated with CT measured fatty liver and ALT levels.

Variants of the ERLIN1-CHUK-CWF19L1 are associated with the joint effect of steatosis (FL $\geq 40 \mathrm{HU}$ ) and inflammation (ALT $\geq 40 \mathrm{U} / \mathrm{L}$ ).

Variants of the ERLIN1-CHUK-CWF19L1 gene cluster may aid in understanding the correlated architecture between liver fat deposition and inflammation process. 


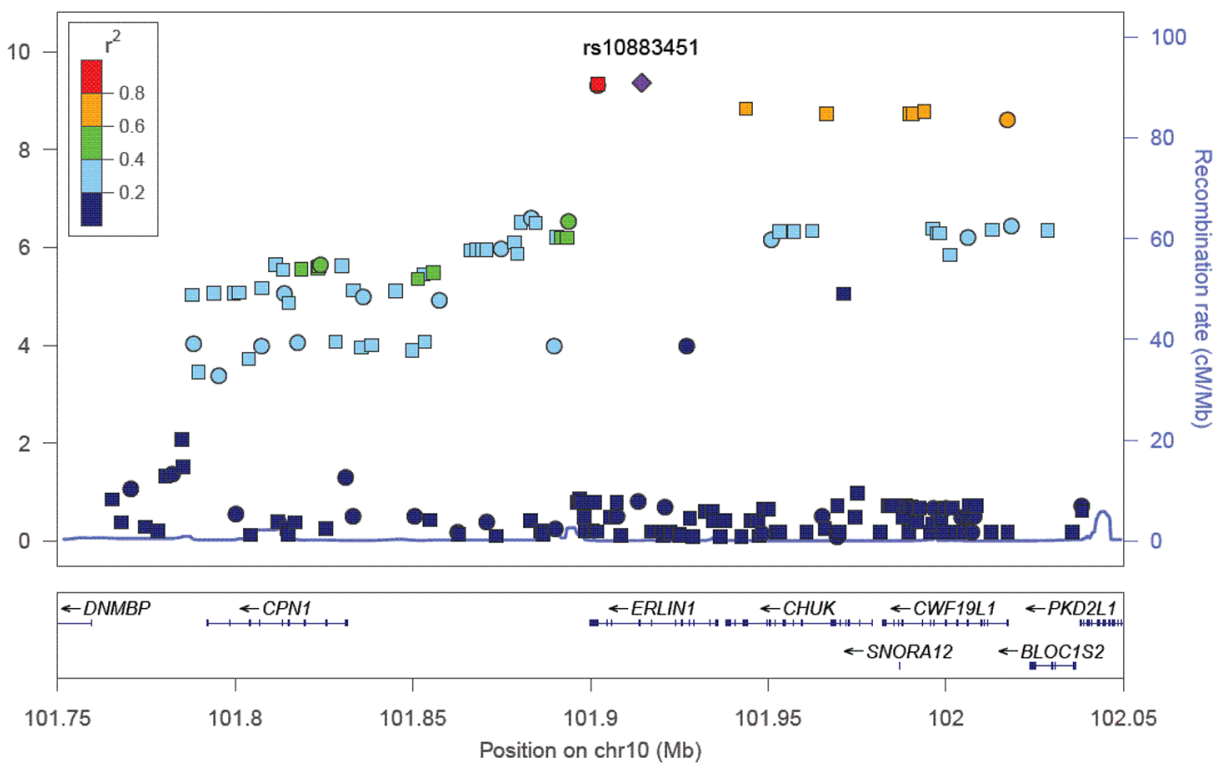

Figure 1.

Regional plot of ERLIN1-CHUK-CWF19L1 gene cluster based on FamHS imputed genotype data. The CMA - $\log \mathrm{P}$ value for correlated FL and ALT pair is represented in the ordinate axis, while the chromosome 10 position in Megabase $(\mathrm{Mb})$ using build 35 is represented in the abscissa axis. The bottom panels give the relative positions of genes in the locus. Purple diamond indicates the effect of rs10883451 with the correlated FL and ALT pair. Each circle indicates a SNP with the color of the circle representing the correlation $\left(\mathrm{r}^{2}\right)$ between that SNP and rs10883451. Blue line indicates estimated recombination hotspots in HapMap. 
Table 1

Characteristics of phenotypes in the FamHS

\begin{tabular}{lllll}
\hline & Number & Mean \pm SD & Minimum & Maximum \\
\hline Sample (\% of females) & $2,705(55 \%)$ & & & \\
Age (years) & 2,705 & $57.1 \pm 13.3$ & 30.0 & 93.0 \\
Alcohol intake (drinks/week) & 2,705 & $3.0 \pm 7.1$ & 0 & 171 \\
CT liver attenuation $(\mathrm{HU})$ & 2,597 & $59.28 \pm 11.02$ & -5.8 & 88.6 \\
ALT (U/L) & 2,584 & $21.1 \pm 13.3$ & 4.0 & 241.0 \\
Triglycerides (mg/dL) & 2,654 & $144.1 \pm 93.9$ & 24.0 & $1,037.0$ \\
Waist circumference $(\mathrm{cm})$ & 2,705 & $98.8 \pm 16.3$ & 58.0 & 187.0 \\
Body mass index $\left(\mathrm{kg} / \mathrm{m}^{2}\right)$ & 2,705 & $28.9 \pm 5.7$ & 14.4 & 57.6 \\
Severe obesity $\left(\mathrm{N}_{\text {Total }} ; \%\right.$ of affected) & $338(2,705 ; 12.5 \%)$ & & & 66.4 \\
HOMA-IR & 2,613 & $2.8 \pm 2.9$ & 0.3 & \\
Diabetes $\left(\mathrm{N}_{\text {Total }} \%\right.$ of affected) & $362(2,705 ; 13.4 \%)$ & & & \\
\hline
\end{tabular}

HOMA-IR: Homeostatic Model Assessment (fasting glucose (mmol/L)*fasting insulin (mU/L)/22.5);

Diabetes: taking hyperglycemic medication and/or fasting glucose $\geq 126 \mathrm{ml} / \mathrm{dl}$;

Severe obesity: BMI $\geq 35.0 \mathrm{~kg} / \mathrm{m}^{2}$ 
